\title{
Neural Correlates of Individual Variability in Fear Extinction
}

\author{
Francisco Sotres-Bayon, ${ }^{1}$ Kevin A. Corcoran, ${ }^{1}$ Jamie Peters, ${ }^{1}$ and Demetrio Sierra-Mercado ${ }^{2}$ \\ ${ }^{1}$ Department of Psychiatry, University of Puerto Rico School of Medicine, San Juan, Puerto Rico 00936-5067, and ${ }^{2}$ Doctoral Program in Biomedical \\ Sciences, Department of Physiology, Ponce School of Medicine, Ponce, Puerto Rico 00732-7004 \\ Review of Hefner et al. (http://www.jneurosci.org/cgi/content/full/28/32/8074)
}

People with post-traumatic stress disorder (PTSD) have difficulty recovering from traumatic events. Although many people are exposed to traumatic events, only a minority of trauma victims subsequently develops PTSD. Such individual differences are often overlooked in animal models, which emphasize group averages and central tendencies. However, understanding the individual differences in animals' responses to traumatic stimuli could be the key to treating and even preventing anxiety disorders like PTSD.

The failure to recover from trauma exposure is thought to reflect deficient extinction of learned fear responses. Fear extinction in rodents provides a useful model to study the behavioral and neural processes underlying PTSD. In fact, research on fear extinction has attracted increasing interest because a major approach to the treatment of these disorders involves promoting extinction (for review, see Milad et al., 2006). Fear extinction is characterized by a progressive reduction in conditioned fear responses with repeated, non-reinforced presentations of the conditioned stimulus. Reduction of fear responses after extinction is thought not to reflect erasure of the original fear memory but rather the retrieval

Received Sept. 12, 2008; revised 0ct. 10, 2008; accepted 0ct. 10, 2008.

We thank Dr. Gregory J. Quirk for discussion and comments on a previous version of this manuscript.

Correspondence should be addressed to Dr. Francisco Sotres-Bayon, Department of Psychiatry, University of Puerto Rico School of Medicine, P.O. Box 365067, San Juan, Puerto Rico 00936-5067. E-mail: fsotresb@gmail.com.

D0I:10.1523/JNEUROSCI.4373-08.2008

Copyright $\odot 2008$ Society for Neuroscience $\quad 0270-6474 / 08 / 2812147-03 \$ 15.00 / 0$ of a newly formed inhibitory memory. This inhibition of fear responses is mediated by interactions between the ventral medial prefrontal cortex and the amygdala (for review, see Sotres-Bayon et al., 2004; Quirk and Mueller, 2008). Successful extinction retrieval requires activity in the infralimbic subregion of ventral medial prefrontal cortex, which suppresses conditioned increases in amygdala activity, thus subsequently reducing fear responses. Consequently, failure to retrieve extinction, as may occur in PTSD, is thought to reflect a lack of infralimbic cortex suppression of amygdala activity, leading to persistent fear responses.

As in most behavioral research, studies on fear extinction use averaged data from sample populations to extrapolate conclusions about the general population. However, this approach ignores the differences between individuals that are exposed to similar traumatic events, yet have differential outcomes with respect to the development of anxiety disorders. In rodents, as in human trauma victims, there is naturally occurring variability in extinction learning (Bush et al., 2007). For example, Burgos-Robles et al. (2007) observed intrastrain variability in rats' ability to retrieve extinction (separate subgroups of rats were either successful or unsuccessful at retrieving extinction). Once these subgroups were identified, the authors were able to retroactively correlate successful extinction retrieval with higher NMDA receptor-dependent bursting in neurons of the infralimbic cortex.

In a recent study published in The
Journal of Neuroscience, Hefner et al. (2008) exploited interstrain differences in mice to identify neural correlates underlying extinction failure and success. They first isolated a mouse strain that is deficient at extinguishing conditioned fear. After a test for extinction learning, Hefner et al. (2008) measured immunoreactivity of the activity-dependent immediate early genes $c$-Fos and Zif268 to identify different patterns of neuronal activity in prefrontal and amygdala extinction circuit within the extinction-resistant (129S1) and normal (C57BL/6J) mouse strains. Consistent with the involvement of infralimbic cortex in successful extinction retrieval, they found that the extinction-resistant strain had lower $c$-Fos and Zif268 activity relative to controls. Moreover, they found that the magnitude of both $c$-Fos and Zif268 immunoreactivity in infralimbic cortex was positively correlated with the degree of extinction retrieval.

Unlike the relatively straightforward patterns of immediate early gene activity in infralimbic cortex, strain differences observed in amygdala activity are more difficult to interpret. Hefner et al. report that the extinction-resistant mice had decreased immediate early gene immunoreactivity in the lateral and basal subnuclei of the amygdala, whereas immunoreactivity in intercalated cell masses and in the central nucleus of the amygdala was increased relative to controls. Increased central amygdala activity in extinctionresistant mice is consistent with the high levels of freezing observed in this strain throughout extinction, as this region is 
necessary for the generation of conditioned fear responses. However, activity in lateral and basal amygdala is also a general requirement for fear expression, and activity in intercalated cells is believed to inhibit fear responses. Thus, the pattern of immediate early gene immunoreactivity in extinction-resistant mice (decreased in lateral and basal amygdala, increased in intercalated cells) is difficult to reconcile with current models of fear and extinction.

In the months since the study of Hefner et al. (2008) went to press, three important studies addressing the roles of different amygdala subnuclei (basal amygdala, lateral amygdala, and intercalated cells) in fear extinction have been published. Here, we describe a model incorporating the results reported by Hefner et al. (2008), in light of these new findings.

In the first of these studies, Herry et al. (2008) identified populations of basal amygdala neurons that are selectively active during high fear ("fear neurons") or low fear ("extinction neurons") states. During extinction retrieval, fear neurons competed with extinction neurons within the basal amygdala for control of central amygdala output. Failure to recruit the extinction neurons in the extinctionresistant strain would then account for the observed decreases in c-Fos and Zif268 expression within this nucleus. Hence, the fear neurons would win the tug-of-war against the extinction neurons and promote fear in the extinction-resistant strain. Because both fear and extinction neurons are putative glutamatergic projection neurons, the fear neurons may promote fear by direct excitation of central amygdala, whereas the extinction neurons may reduce fear via feedforward inhibition of central amygdala through projections to intercalated cells or an indirect projection through infralimbic cortex (Fig. 1).

The second study that may help interpret the findings of Hefner et al. (2008) provides evidence for the involvement of lateral amygdala neurons in extinction as well as fear expression. Jüngling et al. (2008) found that the newly discovered neuropeptide $S$ facilitates fear extinction by enhancing excitatory neurotransmission at lateral amygdala-intercalated cell synapses. Intercalated cells are an inhibitory cluster of cells capable of gating information flow within the amygdala and are believed to be the intermediate by which infralimbic cortex suppresses amygdala output to promote fear extinction (for re-

a

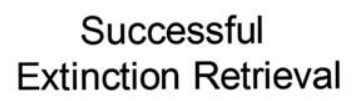

b Unsuccessful
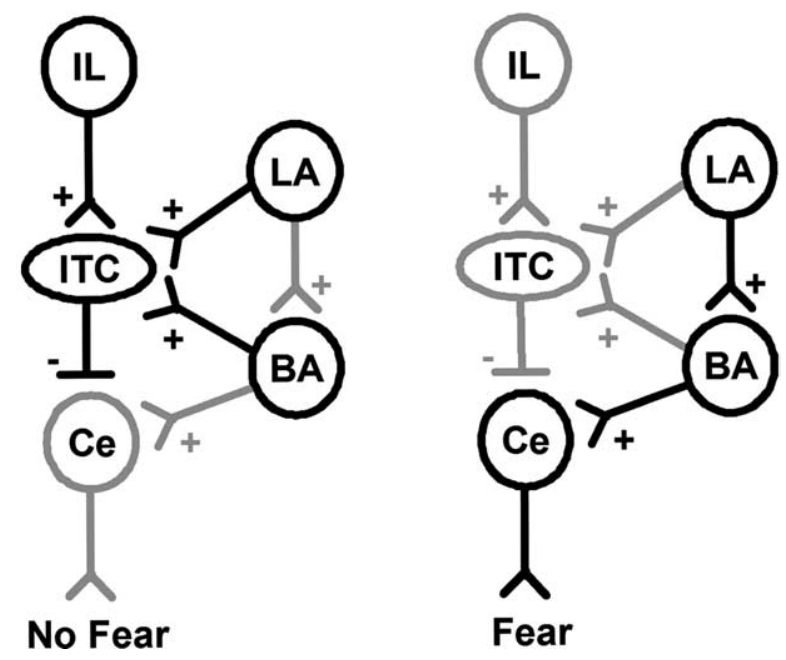

Figure 1. Model for neural correlates of individual variability in fear extinction retrieval. $\boldsymbol{a}$, Extinction retrieval involves increased activity (black) in the infralimbic (IL) prefrontal cortex and extinction neurons in lateral amygdala (LA) and basal amygdala (BA). These amygdala extinction neurons may project to intercalated neurons (ITCS) but not to central amygdala (Ce). The distributed IL-LA/BA "extinction circuit" may orchestrate excitation of inhibitory intercalated amygdala neurons. Activity in ITC suppresses central amygdala and therefore results in suppressed fear responses. $\boldsymbol{b}$, In contrast, in those individuals that are not able to retrieve extinction memory (e.g., those individuals that develop PTSD), the extinction circuit is compromised (decreased activity, gray). Amygdala fear-specific cells, that project to Ce but not ITCs, excite Ce leading to fear expression. In these PTSD-like individuals, the "fear circuit" is more active than the extinction circuit when the fear is no longer appropriate. For simplicity, IL to BA projections that may trigger extinction neurons in BA are not shown.

view, see Paré et al., 2004; Quirk and Mueller, 2008). Thus, the findings of Jüngling et al. (2008) are consistent with a role for lateral amygdala and intercalated cells in promoting extinction. Further, these findings suggest that lateral amygdala neurons that project to intercalated cells may represent a population of neurons that are selectively recruited to suppress fear (Fig. 1a). Hence, the decrease in lateral amygdala Zif268 expression observed by Hefner et al. (2008) is consistent with an extinction-resistant phenotype, but makes the observed increased Zif268 expression in intercalated neurons of the extinction-resistant strain difficult to reconcile with the putative involvement of intercalated cells in promoting extinction.

Until recently, the role for intercalated cells in fear extinction was merely hypothetical and had not been directly tested. Likhtik et al. (2008) resolved this issue by demonstrating that a novel toxin that selectively damages intercalated neurons impairs fear extinction. This represents the strongest evidence to date that activity in intercalated cells promotes extinction. Converging input to intercalated cells from infralimbic cortex, lateral amygdala, and basal amygdala extinction neurons may orchestrate the reduction of fear responses by reducing central amygdala ac- tivity and subsequent fear responses (Fig. 1). That Hefner et al. (2008) show an unexpected positive correlation between Zif268 expression in intercalated cells and fear expression during extinction retrieval is a point of divergence from working models of extinction circuitry and requires further investigation. As suggested by Hefner et al. (2008), one possibility for this divergence is that two functionally different intercalated cell subpopulations mediate fear expression and fear suppression (for further discussion on this possibility, see Paré et al., 2004). The increased Zif268 expression observed in extinctionresistant mice may have reflected activity only in the former. Notwithstanding the difficulty to reconcile their intercalated cell finding, the main conclusion from Hefner et al. (2008) is that resistance to extinction is associated with abnormal recruitment of the infralimbic cortex-amygdala circuits that mediate fear inhibition.

The results of Hefner et al. (2008), combined with these three recent studies, suggest that successful extinction retrieval is orchestrated by activity in infralimbic cortex, lateral amygdala projections to intercalated cells, and basal amygdala extinction neurons, which in turn leads to decreased central amygdala activity and 
diminished fear responses. The opposite pattern of activity in this circuit appears to occur in individuals that fail to extinguish, resulting in increased central amygdala activity and increased fear responses (Fig. 1). It is important to note, however, that Hefner et al. (2008) focused on extinction-related immediate early gene activity after extinction retrieval but not before or after extinction training. Further studies are necessary to contrast strain differences in ventral medial prefrontal cortex and amygdala function during other stages of extinction learning (i.e., acquisition and consolidation). Nonetheless, this extinction circuit presents a reasonable target for pharmacological and genetic interventions for the treatment of PTSD. Moreover, identifying a naturally occurring extinction-resistant mouse strain provides a unique opportunity to identify neural or genetic markers that predispose individuals to extinction failure. Targeting these markers will allow us to predict susceptibility and consequently provide tools to prevent PTSD in humans.

\section{References}

Burgos-Robles A, Vidal-Gonzalez I, Santini E Quirk GJ (2007) Consolidation of fear extinction requires NMDA receptor-dependent bursting in the ventromedial prefrontal cortex. Neuron 53:871-880.

Bush DE, Sotres-Bayon F, LeDoux JE (2007) Individual differences in fear: isolating fear reactivity and fear recovery phenotypes. J Trauma Stress 20:413-422.

Hefner K, Whittle N, Juhasz J, Norcross M, Karlsson RM, Saksida LM, Bussey TJ, Singewald N, Holmes A (2008) Impaired fear extinction learning and cortico-amygdala circuit abnormalities in a common genetic mouse strain. J Neurosci 28:8074-8085.

Herry C, Ciocchi S, Senn V, Demmou L, Müller C, Lüthi A (2008) Switching on and off fear by distinct neuronal circuits. Nature 454:600-606.
Jüngling K, Seidenbecher T, Sosulina L, Lesting J, Sangha S, Clark SD, Okamura N, Duangdao DM, Xu YL, Reinscheid RK, Pape HC (2008) Neuropeptide S-mediated control of fear expression and extinction: role of intercalated GABAergic neurons in the amygdala. Neuron 59:298-310.

Likhtik E, Popa D, Apergis-Schoute J, Fidacaro GA, Paré D (2008) Amygdala intercalated neurons are required for expression of fear extinction. Nature 454:642-645.

Milad MR, Rauch SL, Pitman RK, Quirk GJ (2006) Fear extinction in rats: implications for human brain imaging and anxiety disorders. Biol Psychol 73:61-71.

Paré D, Quirk GJ, Ledoux JE (2004) New vistas on amygdala networks in conditioned fear. J Neurophysiol 92:1-9.

Quirk GJ, Mueller D (2008) Neural mechanisms of extinction learning and retrieval. Neuropsychopharmacology 33:56-72.

Sotres-Bayon F, Bush DE, LeDoux JE (2004) Emotional perseveration: an update on prefrontal-amygdala interactions in fear extinction. Learn Mem 11:525-535. 